\title{
Spatial Covariance Analysis for Tissue Harmonic Imaging
}

\author{
Che-Chou Shen and Pai-Chi Li \\ Department of Electrical Engineering, National Taiwan University, Taipei, Taiwan, R.O.C.
}

\begin{abstract}
Spatial covariance contains important information in the context of correlation-based correction of sound velocity inhomogeneities. Previous work was primarily based on spatial covariance analysis for linear imaging. In this paper, we extend the analysis to tissue harmonic imaging. Specifically, we investigate effects of the signal-tonoise ratio (SNR) and sound velocity inhomogeneities on spatial covariance. It is shown that linear imaging at $\mathrm{f}_{0}$ and second harmonic imaging at $2 \mathrm{f}_{0}$ have similar spatial covariance at high SNRs, regardless the presence of sound velocity inhomogeneities. At low SNRs, however, spatial covariance of tissue harmonic imaging is significantly affected. It is concluded that the linear signal is more suitable for correlation-based correction of sound velocity inhomogeneities despite the fact that tissue harmonic imaging generally has improved image quality over linear imaging.
\end{abstract}

\section{INTRODUCTION}

A major problem for diagnostic ultrasound systems is the image quality degradation resulting from sound velocity inhomogeneities. Sound velocity inhomogeneities distort both amplitude and phase of the acoustic signal. Many methods have been proposed to restore the degraded resolution [1]. All the methods utilize the correlation function to find time delay errors. Performance of the correlation-based method is determined by spatial covariance. The higher the correlation is, the more accurate time delay estimates can be obtained.

Spatial covariance is described by the van Cittert-Zernike theorem [2]. It is shown that spatial covariance and incident pressure field are Fourier pairs. Consequently, spatial covariance decreases linearly as the distance between two channels increases. With focusing errors, the spatial covariance function becomes narrower since the transmit beam becomes wider. One important property of the van Cittert-Zernike theorem is that the spatial dependence of the spatial covariance function is independent of frequency for a given transmitting aperture. As will be shown later, this property plays a critical role in spatial covariance analysis for tissue harmonic imaging.

Tissue harmonic imaging has proven to provide clinically useful images even on technically difficult bodies [3]. Due to its improved acoustic beam characteristics, it was suggested that the tissue harmonic signal may be combined with the linear signal to improve accuracy of correlationbased estimation of time delay errors [4]. To successfully develop an optimal correction strategy, it is necessary to fully understand characteristics of spatial covariance in tissue harmonic imaging.

\section{EFFECTS OF SNR}

Experiments were conducted to measure spatial covariance using the experimental setup shown in Fig. 1. A gelatin-based ultrasonic phantom with uniform distribution of glass beads (Sigma G4649, St. Louis, MO, U.S.A) was used as a speckle generating object. An arbitrary function generator was used to generate the desired transmit waveform and a power amplifier was used to amplify the transmit waveform and to drive a $3.5 \mathrm{MHz}$ single crystal transducer (Panametrics V381, Waltham, MA, U.S.A). The transducer had a diameter of 19 $\mathrm{mm}$ and was geometrically focused at $70 \mathrm{~mm}$.

The scattered signals were received by a separate

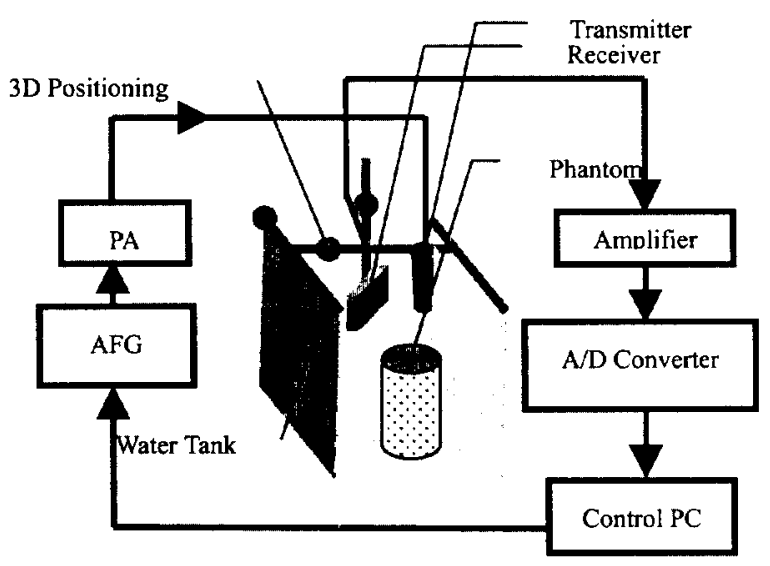

Figure 1: Schematic diagram of the spatial covariance experimental setup. 


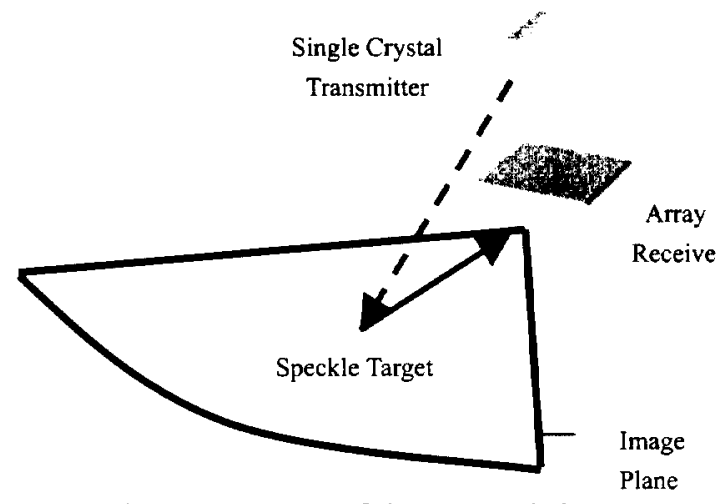

Figure 2: Setup of the transmitting and receiving transducers.

array transducer. Setup of the transmit/receive transducers is graphically shown in Fig. 2.

The two transducers were carefully aligned to ensure that signals from the focal zone of the transmitting transducer were received. For linear imaging, a $5 \mathrm{MHz}$ transducer array was used. For second harmonic imaging, a $7 \mathrm{MHz}$ transducer array was used. Note that a three-axis step motor system was used to position the arrays such that the returning echoes could be received at different locations for spatial covariance analysis. The received signal was sent to an ultrasonic receiver. At each measurement point, the acoustic field was measured 16 times for signal averaging. Finally, an analog-to-digital converter with a $20 \mathrm{Msamples} / \mathrm{sec}$ sampling rate and 12-bit resolution was used to sample the signal for off-line signal processing. The entire experimental setup was controlled by a Pentium-class personal computer with HP VEE software.

The spatial covariance analysis in [2] assumes that the SNR is sufficiently high. In general, the covariance decreases with the SNR. Since the tissue harmonic signal is typically lower than the fundamental signal by more than $20 \mathrm{~dB}$ [3], effects of the SNR cannot be ignored. To test this hypothesis, we investigated the spatial covariance with two different SNRs. The SNR was varied by adjusting the peak amplitude of the output of the function generator (from $1 \mathrm{~V}$ to $5 \mathrm{~V}$ ). Fig. 3 and Fig. 4 show the normalized covariance function (i.e., correlation coefficient) at the fundamental frequency and at the second harmonic frequency. The horizontal axis represents the distance between two measurement positions normalized to the transmitting aperture size. The vertical axis denotes the correlation coefficient. The spatial covariance was not affected by the change in SNR in linear imaging. On the contrary, the correlation coefficient of harmonic imaging reduced significantly when the transmit voltage changed from $5 \mathrm{~V}$ to $1 \mathrm{~V}$.

Due to the low correlation at low SNRs, tissue harmonic imaging dose not provide any advantage over linear imaging.

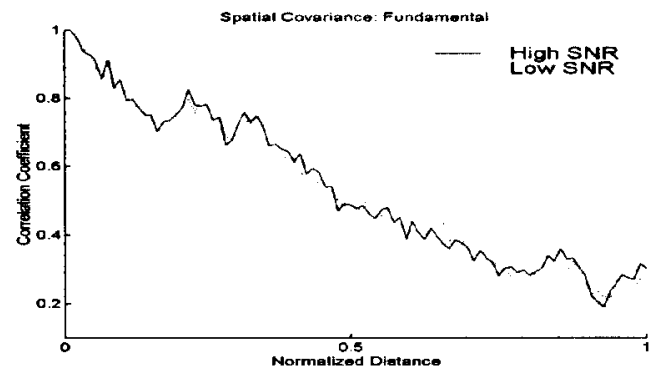

Figure 3: Spatial covariance of the fundamental signal.

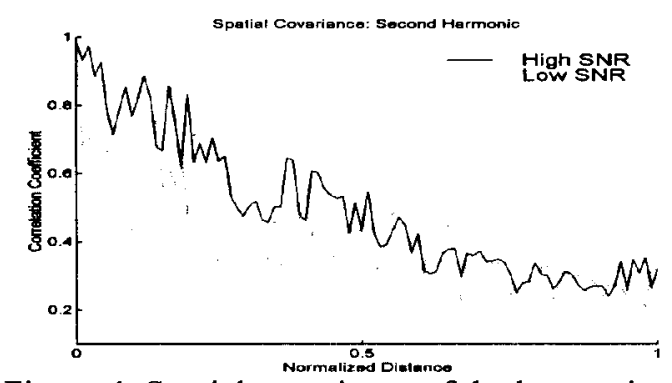

Figure 4: Spatial covariance of the harmonic signal.

\section{EFFECTS OF SOUND VELOCITY INHOMOGENEITIES}

Effects of sound velocity inhomogeneities were also investigated. Peak amplitude of output of the function generator was set to $5 \mathrm{~V}$ to ensure adequate SNR for both fundamental imaging and second harmonic imaging. Fig. 5 shows the measured spatial covariance without sound velocity inhomogeneities. The harmonic covariance is similar to the fundamental covariance except that the harmonic covariance has larger variance. The larger variance is due to the lower SNR of the harmonic signal. Nevertheless, both curves decrease linearly from one to zero as the distance increases from zero to the transmitting aperture size.

The signals received at different positions can be 


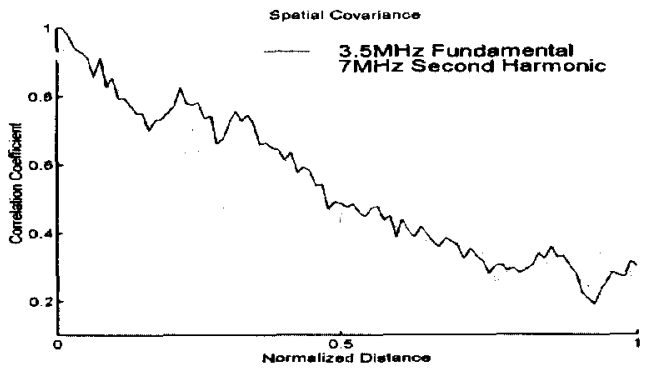

Figure 5: Spatial covariance.

used to form images using the synthetic aperture approach. This is known as the fixed direction transmit and all direction receive focusing in [5]. Fig. 6 shows the fundamental beam and harmonic beam with a $30 \mathrm{~dB}$ display dynamic range. The image is with a sector scan format prior to scan conversion. The horizontal axis is the image range and the vertical axis represents the steering angle.

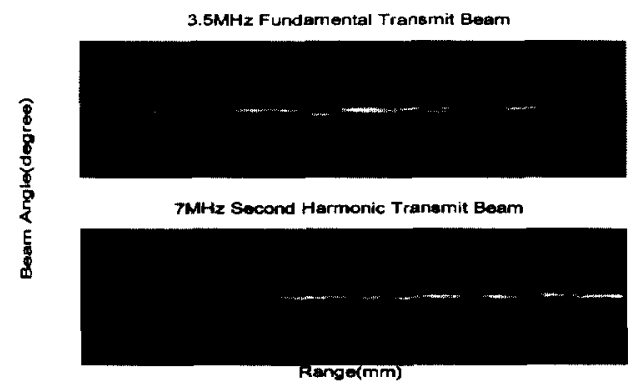

Figure 6:Transmit beam patterns.

Compared to the fundamental beam pattern, the second harmonic beam has a narrower lateral beam width. However, the second harmonic beam does not provide any advantages in the spatial covariance function shown in Fig. 5. As mentioned previously, this is due to the fact that the spatial covariance function is independent of frequency for a given transmitting aperture.

Tissue harmonic imaging is less sensitive to the sound velocity variations present in the body [3]. We conducted experiments to measure the spatial covariance with sound velocity variations by inserting a rubber layer (General Electric RTV511, Waterford, NY, U.S.A.) between the transmitting transducer and the gelatin phantom. The rubber had a sound velocity of $1015 \mathrm{~m} / \mathrm{sec}$ and the averaged thickness was about $1 \mathrm{~cm}$. Spatial sound velocity variations were introduced by the irregular surface. The spatial covariance functions and the corresponding transmit beam patterns are shown in Fig. 7. As shown in the upper panel, the covariance decreased more rapidly than the theoretical curve due to the inserted rubber layer. Nevertheless, the fundamental spatial covariance is generally similar to the second harmonic spatial covariance. The second harmonic beam shown in the lower panel is noticeably narrower than the fundamental beam shown in the upper panel. Again, tissue harmonic imaging does not provide any advantages in accuracy of correlation-based time delay estimation despite the fact that the harmonic beam is narrower than the fundamental beam.

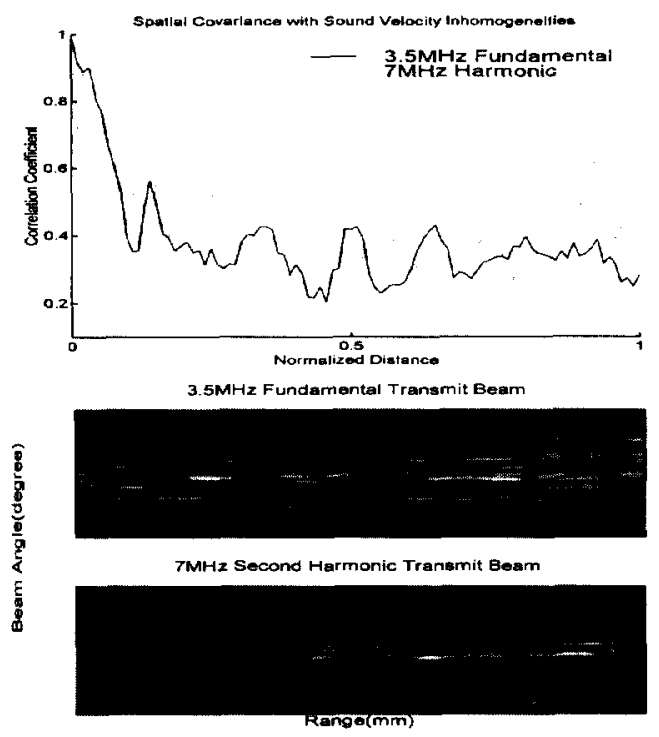

Figure 7: Spatial covariance and beam patterns with sound velocity inhomogeneities.

Spatial covariance of the harmonic signal and the fundamental signal at the same frequency was also evaluated in the presence of sound velocity inhomogeneities. In this case, a $2.5 \mathrm{MHz}$ pulse was transmitted to obtain the second harmonic signal at $5 \mathrm{MHz}$. Another $5 \mathrm{MHz}$ pulse was transmitted for the linear response. The results are compared in Fig 8. It is shown in the upper panel that the $5 \mathrm{MHz}$ harmonic signal has higher covariance than the $5 \mathrm{MHz}$ fundamental signal.

Since the two signals were at the same frequency, the results are consistent with the measured beam patterns shown in the lower panel. 

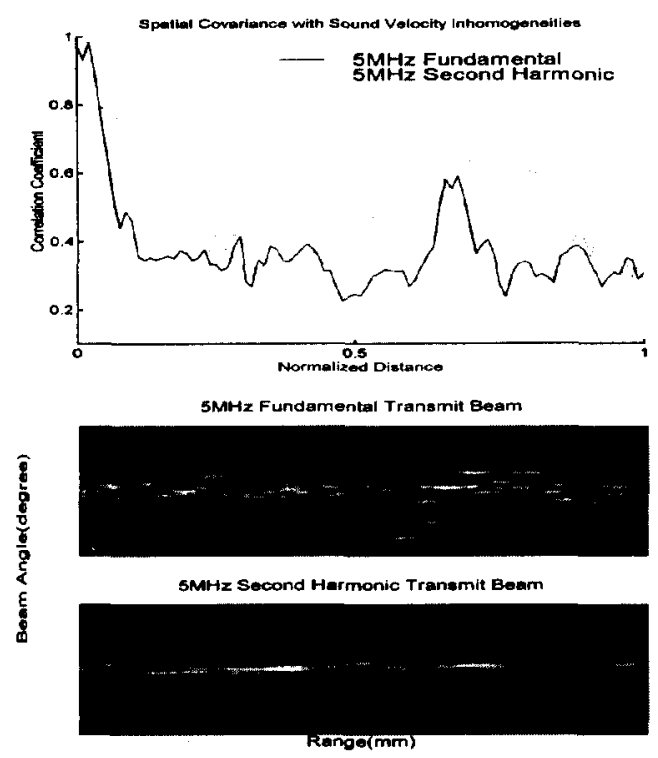

Figure 8: Spatial covariance and beam patterns with sound velocity inhomogeneities.

Finally, Fig. 9 shows the spatial covariance functions for linear imaging at both $3.5 \mathrm{MHz}$ and $5 \mathrm{MHz}$ in the presence of sound velocity inhomogeneities. The $5 \mathrm{MHz}$ array transducer was used to receive returning echoes in both cases. Since the time delay errors were introduced by the same rubber layer, sound velocity inhomogeneities produced small phase errors at the lower frequency and larger phase errors at the higher frequency. Thus, the covariance function at $3.5 \mathrm{MHz}$ declines more slowly than the $5 \mathrm{MHz}$ counterpart. In other words, the lower portion of the received linear signal generally provides more accurate correlationbased estimation results.

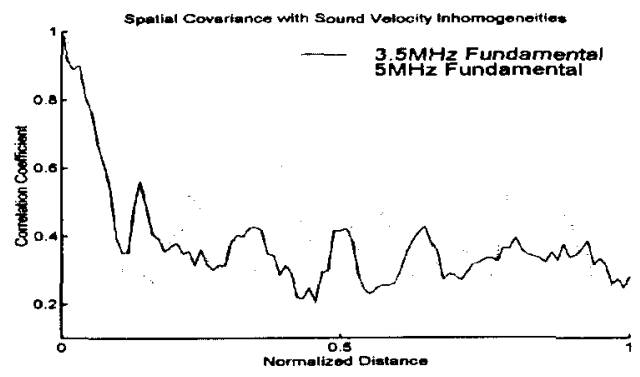

Figure 9: Spatial covariance with sound velocity Inhomogeneities.

\section{CONCLUSIONS}

In this paper, spatial covariance under various conditions was investigated. It was shown that if adequate SNR is available, the spatial covariance functions at both the fundamental frequency and the second harmonic frequency have similar performance. At a lower SNR, however, the covariance between signals received from two different positions decreased and accuracy of the correlation-based estimation may be affected. Since the SNR of tissue harmonic imaging is significantly lower than the SNR of fundamental imaging, the linear signal is preferred for correlation-based correction of sound velocity inhomogeneities. Note that both the fundamental signal at $\mathrm{f}_{0}$ and the second harmonic signal at $2 \mathrm{f}_{0}$ are available for a single firing. Thus, a tissue harmonic imaging system may use the fundamental signal for correlation-based time delay estimation and the second harmonic signal for imaging.

In the presence of sound velocity

inhomogeneities, the spatial covariance function of tissue harmonic imaging becomes higher than that of fundamental if both are at the same frequency (e.g., $f_{0}$ ). However, it requires two firings for each beam since the transmit frequencies are different. Thus, it may result in $50 \%$ frame rate reduction.

Finally, performance of the correlation-based processing can be improved by using a lower frequency portion of the received linear signal. As shown in Fig. 9, the $3.5 \mathrm{MHz}$ covariance is noticeably higher than the $5 \mathrm{MHz}$ covariance. Thus, the lower frequency portion of a received broadband echo can be used to improve accuracy of correlation-based time delay estimation.

\section{REFERENCES}

[1] S. W. Flax and M. O'Donnell, "Phase-aberration correction using signals from point reflectors and diffuse scatterers: basic principles," IEEE Trans. Ultrason., Ferroelect., Freq. Contr., vol. 35, no. 6, pp. 758-767, Nov. 1988.

[2] M. Fink and R, Mallart, "The van Cittert-Zernike theorem in pulse echo measurements," $J$. Acoust. Soc. Amer, vol. 90, no. 5, pp. 2718-2727, Nov. 1991.

[3] T. Christopher, "Finite amplitude distortion-based inhomogeneous pulse echo ultrasonic imaging," IEEE Trans. Ultrason., Ferroelect., Freq. Contr, vol. 44, no. 1, pp. 125-139, Jan. 1997.

[4] D. Langdon, G. Holley, J. Hossack and P.-C. Li, "Ultrasonic Imaging Aberration Correction System and Method", U.S. Patent number 6,023,977, Feb. 2000.

[5] P.-C. Li, S.W. Flax, E.S. Ebbini and M. O'Donnell, "Blocked Element Compensation in Phased Array Imaging", IEEE Transactions on Ultrasonics, Ferroelectrics and Frequency Control, Vol. UFFC-40, No.4, pp. 283-292, July 1993. 